ISSN: $1130-3743$ - e-ISSN: 2386-5660

DOI: http://dx.doi.org/10.14201/teoredu2016282249265

\title{
LA PERSPECTIVA BIOGRÁFICA. EL PROCESO DE CONSTRUCCIÓN DEL SABER PEDAGÓGICO
}

\section{The biographical perspective. The process of construction of pedagogical knowledge}

\section{Perspective biographique. Le processus de construction des connaissances pédagogiques}

\author{
María SOTOS SERRANO*, Carmen LÓPEZ EsteBAN*** y Ana Belén SÁNCHEZ GARCÍA**** \\ * Universidad de Castilla-La Mancha. Facultad de Educación. \\ Departamento de Matemáticas.Albacete.maria.sotos@uclm.es \\ * Universidad de Salamanca. Facultad de Educación. \\ Departamento de Didáctica de las Matemáticas y de las Ciencias Experimentales. \\ C/ Paseo de Canalejas, 169.37008 Salamanca.lopez@usal.es \\ ** Universidad de Salamanca. Facultad de Educación. \\ Departamento de Didáctica, Organización y Métodos de Investigación. \\ C/Paseo de Canalejas, 169.37008 Salamanca.asg@usal.es
}

Fecha de recepción: enero de 2016

Fecha de aceptación: marzo de 2016

RESUMEN

El objetivo principal de este trabajo consiste en analizar si la perspectiva biográfica, mediante los relatos de vida, permite analizar los procesos de construcción del saber pedagógico de las/os docentes, ya que pensamos que la formación docente supera los límites estrictos de los períodos de formación reglada. También se presentan algunos de los objetivos que justifican este tipo de estudios y, finalmente, se defiende la tesis de que la perspectiva biográfica es la que permite cumplir con esos objetivos. Para ello planteamos un estudio de corte fenomenológico sobre el caso de 
M. Antònia Canals, fundamentado en la obtención de datos cualitativos a través de grabaciones de entrevistas abiertas, y a su vez se presenta un método de análisis de 4 fases que cuenta con amplia aceptación en el marco de la investigación social cualitativa.

Palabras clave: historia de vida; análisis cualitativo; formación docente; M. Antònia Canals; Didáctica de las Matemáticas; metodología de investigación.

\section{SUMMARY}

The main goal of this work is to analyze if the biographical perspective, through the real life stories, allows you to analyze the processes of construction of pedagogical knowledge of the teaching, since we think that teacher training exceeds the limits of formal training periods. Some of the objectives that justify such studies are presented and, finally, we defend the thesis that the biographical perspective allows you to meet those objectives. So we present a study of phenomenological court on the case of M. Antònia Canals, based on qualitative data through open interviews recordings, and in turn we present a method of analysis of 4 phases which has wide acceptance in the framework of qualitative social research.

Key words: life history; qualitative analysis; teacher training; M. Antònia Canals; didactic of the mathematics; research methodology.

\section{SOMMARIE}

L'objectif principal de cet article est d'analyser si la perspective biographique, à travers des histoires de vie, permet d'analyser les processus de construction des connaissances pédagogiques des enseignants, puisque la formation des professeurs va bien au-delà des limites imposées par les périodes de la formation officielle réglée. On présente aussi, quelques-uns des objectifs qui justifient ce genre d'études. Finalement on défend la thèse que c'est justement la perspective biographique celle qui permet d'atteindre ces objectifs. Pour cela, nous proposons une étude phénoménologique sur le cas de M. Antònia Canals, basée sur des données qualitatives obtenues au moyen d'entrevues ouvertes enregistrements. Dans le même temps, on présente une méthode d'analyse de 4 phases qui est largement acceptée dans le cadre de la recherche sociale qualitative.

Mots clés: histoire de vie; analyse qualitative; formation des enseignants; M. Antònia Canals; enseignement des mathématiques; de la méthodologie de recherche. 


\section{INTRODUCCIÓN}

Desde la década de los 30 del siglo xx, se han sucedido numerosos trabajos de investigación empírica que han dado lugar a diversos modelos que intentan explicar el comportamiento de la actuación del profesorado en su trabajo en el aula. Es un campo que cuenta con una extensa tradición y cuya importancia para el análisis de los procesos educativos nadie pone en duda.

Hasta los años 70 del pasado siglo, los modelos sobre pensamiento del profesorado estaban excesivamente vinculados a la concepción de la racionalidad técnica, que daba por supuesto que, en su práctica, cualquier profesional utiliza técnicas y procedimientos derivados del conocimiento científico aplicable, mientras que, en esas mismas investigaciones, la realidad práctica del aula se presenta como una situación caracterizada por la complejidad, la incertidumbre, la inestabilidad y el conflicto (Pérez, 1985). Desde esa racionalidad técnica se presuponía al docente como un elector racional que solamente ha de aplicar a las situaciones de enseñanza-aprendizaje aquellos elementos propios de su formación científica, elementos que se intentarán traducir en una batería de variables cuantitativas que permitieran la aplicación de técnicas de análisis estadístico multivariable. En este sentido resulta especialmente ejemplar la crítica realizada por Contreras (1985), que plantea que este tipo de investigaciones mantiene implícitamente un modelo de enseñanza cuya finalidad es el máximo rendimiento del alumno, modelo que también se supone que comparten docentes e investigadores; a la vez que adolecen de un excesivo carácter estático de la investigación, por lo que no se puede analizar cómo la propia práctica docente modifica los esquemas mentales del profesorado.

En definitiva, «no se han desarrollado modelos explicativos de la complejidad de los procesos de enseñanza y aprendizaje porque no se han llegado a considerar cuáles son las características de la complejidad. Y no se ha podido llegar a conocer y comprender la complejidad porque no se ha partido de investigar las características de las situaciones naturales en que se enmarcan las actuaciones docentes y su influencia mutua” (Arnaus, 1993, 20-21).

Es en este terreno de la comprensión de los fenómenos sociales en el que destacan los trabajos de Elbaz, Cladinin y Connelly ${ }^{1}$, que metodológicamente suponen un punto de ruptura respecto a la tradición anterior. El concepto de conocimiento práctico, en el que se incluyen los significados conscientes o inconscientes, personales o sociales, que se expresan en las acciones de una persona (Connelly y Clandinin, 1986), supone la necesidad de plantear nuevas formas de investigación

1. En el comienzo de la década de los ochenta hay otros trabajos que plantean una metodología basada en entrevistas abiertas y observación del trabajo en el aula (JANESICK, 1982; LARSSON, 1984), pero es en los trabajos de Elbaz y Cladinin en donde se desarrolla y conceptualiza una nueva forma de entender la investigación sobre el profesorado, frente a los enfoques cognitivos anteriores. Junto a estas dos autoras también aparece la figura de Connelly, todos ellos miembros del Ontario Institute for Studies in Education de la Universidad de Toronto. 
para poder comprenderlo. "Nuestro interés es reimaginar la epistemología de la enseñanza y así ofrecer una manera alternativa de ver las clases. Hemos identificado esta manera de investigación de las clases como una perspectiva narrativa. La perspectiva narrativa se preocupa de las historias personales de los participantes, enmarcadas dentro de la historia social de las escuelas y de la enseñanza" (Connelly y Clandinin, 1986, 297). Resumiendo un amplio conjunto de investigaciones afines, la década de los ochenta puede entenderse como la ruptura con los modelos precedentes de investigación del profesorado y la consolidación de una nueva perspectiva científica.

Si hemos señalado la obra de Elbaz como un punto de ruptura con la tradición anterior, también el uso de una perspectiva biográfica supone una novedad en esta nueva forma de abordar el estudio sobre el profesorado. Evidentemente no se trata de una ruptura similar, ya que se mantienen los principios epistemológicos de las investigaciones cualitativas, pero se amplía el campo de estudio más allá del análisis de la práctica en el aula, que hasta ahora se solía realizar mediante algunas observaciones que podían prolongarse, como máximo, a todo un curso escolar; así como algunas entrevistas abiertas que pretendían indagar sobre las creencias del docente, muchas veces sin abordar el cambio y la evolución de dichas creencias a lo largo del tiempo.

Es en el campo de la sociología en el que comienzan a utilizarse, a comienzos del siglo Xx, diferentes tipos de documentos personales como fuentes de datos para una serie de investigaciones que guardan estrecha relación con el trabajo que se desarrollaba en el ámbito de la antropología ${ }^{2}$, aunque su desarrollo posterior no gozó de continuidad. «La historia contada por escrito, en artículos y manuales sobre la metodología biográfica, resume el paso del tiempo refiriéndose, reiteradamente, a su uso primero (en la época del nacimiento y desarrollo del método, años veintetreinta), desuso posterior (años cuarenta-cincuenta) y renacimiento con intereses renovados de los años sesenta, setenta y ochenta» (Valles, 1997, 250).

\section{LA PERSPECTIVA BIOGRÁFICA Y SU PROYECCIÓN AL CAMPO DE LOS ESTUDIOS SOBRE PROFESORADO}

Respecto a los estudios sobre el profesorado, esta tendencia llega algunos años después, pues incluso las investigaciones sobre creencias y concepciones del docente se realizaban desde un punto de vista estático, soslayando los cambios y la evolución de dichas creencias y concepciones. En 1981, Goodson recomendaba los métodos biográficos para la investigación sobre el profesorado, y es a partir de la segunda mitad de los años ochenta y principios de los noventa cuando se

2. En este trabajo no se pretende realizar un recorrido por la historia de la perspectiva biográfica en las ciencias sociales, pero para las argumentaciones históricas que aquí se exponen se han consultado las obras de Balan (1974) y de VALLES (1997). 
produce una cierta expansión en el terreno de la pedagogía ${ }^{3}$. Hasta esas fechas, las investigaciones sobre el profesorado solían centrarse en el período de formación inicial, ya que se pretendía averiguar cuáles eran las mejores condiciones para dicha formación, pues de ella dependía toda su trayectoria profesional posterior. "Mucho se ha escrito e indagado sobre el cómo y el porqué de la resistencia del profesor a los cambios, y uno de los hallazgos en este campo ha sido que no puede mirarse al profesor sólo en el momento biográfico en el que se encuentra, sino que es necesario recorrer su trayectoria [...] para comprender el lugar en el que se sitúa y su disposición para la innovación y el cambio» (Hernández, 2004, 11).

La obra de Goodson (2004) es la que mejor resume este nuevo panorama en los estudios sobre el profesorado desde la perspectiva biográfica ${ }^{4}$. En el prefacio a la edición española indica que «los estudios de historia de vida sobre la enseñanza contribuyen a la producción de una amplia gama de saberes profesionales que emergen del propio docente» $(2004,37)$, y continúa con una cuestión crucial en el planteamiento epistemológico de cualquier investigación, la que tiene que ver con el porqué y el para qué de cada investigación:

Nuestra intención, lejos de buscar un «cierre» académico, es [...] la de crear espacios vitales para la reflexión y el comentario. Este tipo de trabajo tiene como objetivo desarrollar nuevas estrategias para que los docentes analicen y reflexionen sobre su vida y su labor profesional de tal modo que puedan dar respuestas más profundas y poderosas frente al mundo socialmente construido de la educación» (Goodson, 2004, 39).

Lo que remite directamente a uno de los pilares fundamentales de la investigación cualitativa en ciencias sociales: la consideración de los objetos de estudio no como objetos a estudiar, sino como lo que realmente son, sujetos reflexivos cuyo papel en la investigación no se puede limitar al mero ofrecimiento de datos a otro sujeto, el investigador, que nunca debiera situarse en un plano superior al de los sujetos investigados 5 .

Esta cuestión tiene más importancia de la que habitualmente se le da en los ámbitos académicos de investigación, ya que las opciones metodológicas implican

3. Nos referimos a autores como R. Butt, D. Raymond, P. Woods, P. Sikes, M. Huberman e I. Goodson, entre otros.

4. La obra original en inglés es de 1992, por lo que la traducción al castellano doce años después puede significar el retraso añadido del caso español. La tesis doctoral de ARNAUs (1993) puede señalarse como el momento en que esta tradición metodológica entra en el ámbito académico de la pedagogía en la universidad española.

5. Esta consideración de los sujetos (investigadores e investigados) por parte de la investigación social cualitativa guarda una estrecha relación con lo que P. Freire denominó concepción liberadora de la educación, en donde no hay una línea de demarcación entre educador y educando, sino educador-educando y educando-educador. De hecho, las implicaciones de la concepción liberadora de la educación y de la manera de abordar la investigación social como investigación participativa también resultan similares. 
opciones epistemológicas con efectos directos en el terreno de la ética y la política. Así, «a fuerza de omitir las voces de los profesores y profesoras corrientes, lo que hace en realidad la bibliografía sobre las carreras profesionales de los docentes es silenciarlas. [...] Teóricamente lo que se desarrolla es una visión instrumental del profesorado, reduciéndolo a la categoría de objeto que puede ser manipulado para alcanzar determinados fines» (Casey, 2004, 246).

Pero en este recorrido histórico sobre las investigaciones sobre el profesorado, lo que queremos destacar es que esta génesis histórica no es sólo un recorrido por las diversas metodologías que se han ido utilizando en el estudio del profesorado, sino que detrás de cada metodología existen una serie de elementos epistemológicos que la sustentan 6 , y que estos elementos son la primera cuestión a la que tiene que responder cualquier intento de investigación que quiera ser realizado con honestidad.

Compartimos el criterio de Bertaux acerca de la capacidad que tiene el estudio de las trayectorias personales para "captar mediante qué mecanismos y qué procesos ciertos individuos han terminado encontrándose en una situación dada y cómo tratan de acomodarse a esa situación» (Bertaux, 2005, 19). Pero para esto hay que considerar estas trayectorias como sistemas complejos en donde se producen interrelaciones entre múltiples aspectos (personales y sociales), y en donde muy pocas veces la causalidad lineal (entendida como la causa eficiente aristotélica) sirve para explicar dichas trayectorias.

Precisamente, la perspectiva de la investigación biográfica permite establecer conexiones hacia atrás, ya que uno de los puntos fuertes de la metodología biográfica es que «el carácter retrospectivo, longitudinal, de la información recabada permite un conocimiento, a fondo, de la cronología y los contextos de surgimiento y desarrollo de la interacción social y de los puntos de vista de los individuos" (Valles, 1997, 252). De ahí la necesidad de esta estrategia metodológica para poder descubrir el proceso de construcción del saber pedagógico de cualquier docente.

Tardif (2004), en su teoría de los saberes docentes, plantea el concepto de saber pedagógico a partir de la reflexión compartida sobre los saberes prácticos. Mientras que los saberes prácticos son saberes específicos desarrollados por el

6. La ontología y la epistemología remiten a la cuestión de qué es lo que está al alcance del conocimiento humano y de cómo poder acceder a ese conocimiento. No en vano, el renacimiento de la investigación cualitativa en las ciencias sociales viene del llamado giro interpretativista que se gesta en la década de los sesenta del siglo xx, curiosamente una década en la que se desarrollan determinados fenómenos de rechazo y revuelta contra el modelo dominante de las sociedades occidentales.

Junto a esto, y con el mismo nivel de importancia, también hay que plantearse el porqué y el para qué de nuestro trabajo investigador, lo que lleva directamente a las consideraciones éticas y políticas de la investigación social. Así, tampoco es casual el importante papel que juega la teoría crítica de la Escuela de Fráncfort en ese renacimiento de las investigaciones cualitativas, ya que «lo que el positivismo consagra es la no racionalidad de la esfera de los fines, y lo que la teoría crítica reivindica es justamente la restitución de los fines del hombre al ámbito de la racionalidad, esto es, de la ciencia” (BELTRÁN, 1992, 28). 
docente a partir de su trabajo cotidiano y del conocimiento de su medio, «el saber pedagógico se presenta como una construcción propia dentro del sujeto, que lleva a cabo como resultado de las interacciones entre sus disposiciones internas y el contexto cultural y social de manera activa y participativa, que le permite organizar, interpretar y reestructurar el conocimiento con la experiencia, los saberes previos y la información que de diversas fuentes recibe» (Díaz, 2005, 6).

\section{DiseÑO DE INVESTIGACIÓN}

La propuesta metodológica que se presenta en este trabajo consiste en el uso de los relatos de vida para el análisis de las creencias del profesorado sobre su práctica pedagógica y las posibles proyecciones sobre los saberes docentes, al objeto de generar contextos de reflexión sobre la propia praxis que constituye el objetivo principal de esta investigación. Tal vez el término más conocido sea el de historia de vida, pero hay que señalar que Denzin (1970) distinguió entre life story y life history. En el primer caso se refería a la historia de la vida de una persona tal y como la cuenta dicha persona, mientras que en el segundo caso se refería a la historia de una persona realizada a partir no sólo de su propio relato, sino también mediante otro tipo de documentos. Esta distinción está totalmente admitida en la comunidad científica y, para evitar la confusión de la traducción literal de los términos anglosajones, en castellano se suele utilizar la propuesta de Bertaux de distinguir entre historia de vida y relato de vida.

Estos planteamientos son los que hemos utilizado para realizar una investigación de caso único, mediante la construcción del relato de vida de Maria Antònia Canals (Sotos, 2015), en la que tratamos de conocer e interpretar elementos actitudinales inherentes a la propia trayectoria vital de Maria Antònia Canals. Por tanto, la metodología utilizada es de tipo cualitativo; puesto que las creencias y actitudes y su proyección a la praxis docente no son directamente observables, sino inferidas a partir de la conducta verbal o no verbal del sujeto objeto de estudio. Por esta razón, en el proceso de recolección de datos acumulados, para su análisis, destacan los discursos obtenidos mediante entrevistas abiertas (Aguilar, 1999), tanto para la persona cuyo relato de vida se va a construir, como para aquellas otras que, por su relación con la primera, sean relevantes como fuentes adicionales de datos. Esta relevancia se refiere, básicamente, a dos cuestiones: por un lado, para completar y corregir, mediante un sencillo mecanismo de triangulación, el relato de vida y, por otro lado, para descubrir, en las discrepancias, los procesos de interpretación que cada sujeto hacemos cuando reelaboramos nuestra biografía.

Junto a las entrevistas, también se analizaron los documentos relevantes disponibles. Los documentos con datos primarios tienen la misma función correctora que señalamos en las entrevistas a terceras personas, mientras que los documentos con datos secundarios, en caso de que existan, ya incorporan análisis previos realizados por otros investigadores que cuentan con cierta relevancia previa. 
MARÍA SOTOS SERRANO, CARMEN LÓPEZ ESTEBAN Y ANA BELÉN SÁNCHEZ GARCÍA LA PERSPECTIVA BIOGRÁFICA. EL PROCESO DE CONSTRUCCIÓN DEL SABER PEDAGÓGICO

\subsection{Metodología}

Para el análisis de datos hemos utilizado el software ATLAs.ti v. 6.2.15. Partimos del total de textos grabados y seguimos la idea de Bertaux de hallar la estructura diacrónica de la bistoria reconstruida. Los datos con los que se elabora un relato de vida no presentan un discurso perfectamente lineal de la trayectoria de los sujetos, por ello es necesario establecer esa estructura diacrónica. A partir de ahí, «el análisis de una entrevista biográfica tiene por objeto explicitar las informaciones y significados pertinentes que en ella se contienen» (Bertaux, 2005, 90), y para desarrollar un análisis comprensivo de este tipo de materiales se precisa de imaginación y rigor. "La prioridad es de la imaginación, puesto que se trata de imaginar, es decir, crearse una representación (primero mental y después discursiva) de las relaciones y procesos que han dado origen a los fenómenos de los que hablan los testigos [...]. La reconstrucción de la estructura diacrónica no es sólo una operación técnica; esa reconstrucción prepara al analista para la búsqueda de vías de causalidad secuencial, de procesos en cadena” (Bertaux, 2005, 91).

Dicha reconstrucción diacrónica no sólo permite establecer el relato biográfico en sí mismo, sino que plantea también las relaciones que permitieron los diferentes procesos que se señalan en el relato. Para esto, se sigue un proceso de tratamiento analítico que mostramos en el apartado siguiente que puede ser esquematizado en cuatro fases, y suele ser el más utilizado en el análisis de las entrevistas en profundidad (Valles, 1997) ${ }^{7}$.

\subsection{Instrumentos de recogida de datos}

En el caso concreto de la tesis doctoral realizada sobre Maria Antònia Canals (Sotos, 2015), la información procede de fuentes de investigación primarias y los instrumentos que sirvieron para recoger datos de dicha información fueron, en primer lugar, la entrevista abierta y otras fuentes de investigación como cartas y correos electrónicos. También se utilizaron fuentes de investigación secundarias (10 libros y artículos publicados), pero estas no se codificaron mediante ATLAs.ti.

Después de realizar todo el trabajo de campo, disponíamos de las transcripciones de 29 entrevistas (19 con M. Antònia y otras 10 con personas relacionadas con ella), 5 cartas remitidas por ella y otros 5 correos electrónicos de antiguos compañeras/os suyas/os (una alumna y una maestra de Ton i Guida, y 3 personas de grupos de maestras/os organizados por M. Antònia). En total eran casi 350 páginas y para poder manejar con cierta facilidad todo ese material, utilizamos el software ATLAS.ti. El total de unidades analizadas a través de este software ha sido de 13.850 líneas de texto.

7. También hay ejemplos del empleo de este mismo tipo de análisis en investigaciones sobre la enseñanza de las matemáticas (PLASENCIA y otros, 1999). 
Siguiendo a Valles (1997), una vez realizada la compilación de toda la información dentro del programa, se procedió a la codificación de la misma mediante el proceso que se describe a continuación:

(i) Codificación abierta. Con las transcripciones de las entrevistas se comienza un proceso de simplificación de la información que contienen, que consiste en ir delimitando fragmentos en función de los temas, las personas o los lugares que se consideran significativos para el relato biográfico, y a dichos fragmentos se les asignan unos códigos que sirven para clasificarlos. Además, en esta primera fase también se suelen escribir notas explicativas, en donde se formulan algunas hipótesis sobre posibles relaciones entre diferentes códigos, que se retomarán en las dos últimas fases. "El objetivo que se persigue con dicha codificación es abrirse a la indagación. Cualquier interpretación en esta fase pertenece al campo de la improvisación» (Trinidad, Carrero y Soriano, 2006, 48).

Cuando se dispone del suficiente material discursivo (algo habitual en las investigaciones cualitativas) lo normal es que esta primera codificación genere un listado de códigos muy extenso que, para poder analizarlo, conviene reducirlo. Para ello se pueden crear familias de códigos, en donde se agrupan códigos que pertenecen a un mismo campo semántico y a las que se les otorgan nuevos códigos, que serán con los que se trabaje en las siguientes fases.

(ii) Agrupación. Consiste en reunir todos los fragmentos de un mismo código, pero sin perder la referencia del texto del que procede cada uno de ellos.

(iii) Integración local. A partir del trabajo anterior, se procede a interpretar cada una de las agrupaciones por separado, estableciendo qué categorías descriptivas o conceptuales caracterizan mejor cada código. Esta operación es lo que Weiss (1994) denomina integración local.

(iv) Codificación selectiva. Tras la integración local, se trata de averiguar qué código o códigos pueden ser tomados como categorías centrales. Este proceso parte de las relaciones que existen entre los diferentes códigos y permite reducir el número inicial de categorías (Trinidad, Carrero y Soriano, 2006, 51). Otros autores denominan a esta fase integración inclusiva (Weiss, 1994), en la que se organizan todos los códigos alrededor de la secuencia narrativa y explicativa del relato de vida. En ambos casos, de lo que se trata es de descubrir las categorías centrales que están presentes a lo largo del relato de vida, qué relaciones mantienen entre ellas y qué papel desempeñan el resto de categorías en dicha secuencia.

En el caso de Maria Antònia Canals, las categorías centrales que permiten enmarcar su trayectoria son de dos tipos, profesionales (educación y matemáticas) y comportamentales (compromiso e independencia), y es en la propia reconstrucción autobiográfica que realiza Maria Antònia en la que aparecen dichas categorías, ya que «la construcción de una historia de vida es el modo mediante el cual el 
individuo representa aquellos aspectos del pasado que son relevantes para la situación presente [...]. Las historias de vida no son, por tanto, una colección de todos los acontecimientos del curso de la vida individual, sino más bien autoimágenes estructurales» (Kohli, 1993, 177).

\section{Resultados}

De manera general, podemos decir que el paradigma interpretativo en el que se apoya esta investigación nos permitió la descripción ideográfica de las prácticas docentes de M. ${ }^{a}$ Antonia Canals con un alto grado de profundidad, de manera tal que su ideografía pedagógica quedó altamente individualizada. Para acceder a este nivel de profundidad es necesario codificar la información obtenida, teniendo en cuenta que, sobre el total de unidades textuales analizadas que contenían los documentos, es necesario conocer sobre qué aspectos queremos buscar información. Esos aspectos serían las categorías que vamos a utilizar para el análisis posterior, y en este caso fueron: el carácter personal, el entorno familiar, las cualidades personales, la escuela, la universidad, las matemáticas, l'Escola Ton i Guida y el contexto político. Sabiendo que queremos hacer el análisis a partir de esas 8 categorías, comenzamos a asignar códigos a los fragmentos de texto que se consideran pertinentes, mediante lo que se conoce como codificación abierta. En este tipo de codificación, y pese a saber cuáles serían las categorías que nos interesaban, no se parte de un listado de códigos previo, sino que se definen los códigos a medida que se van necesitando.

En la pantalla del programa ATLAS.ti (Imagen 1) se puede apreciar el texto en el panel izquierdo (donde se van seleccionando fragmentos textuales), mientras que el panel derecho es donde van apareciendo los códigos asignados a cada selección de texto. 


\section{IMAGEN 1}

PANTALLA PRINCIPAL DE ATLAS.TI CON UN TEXTO YA CODIFICADO

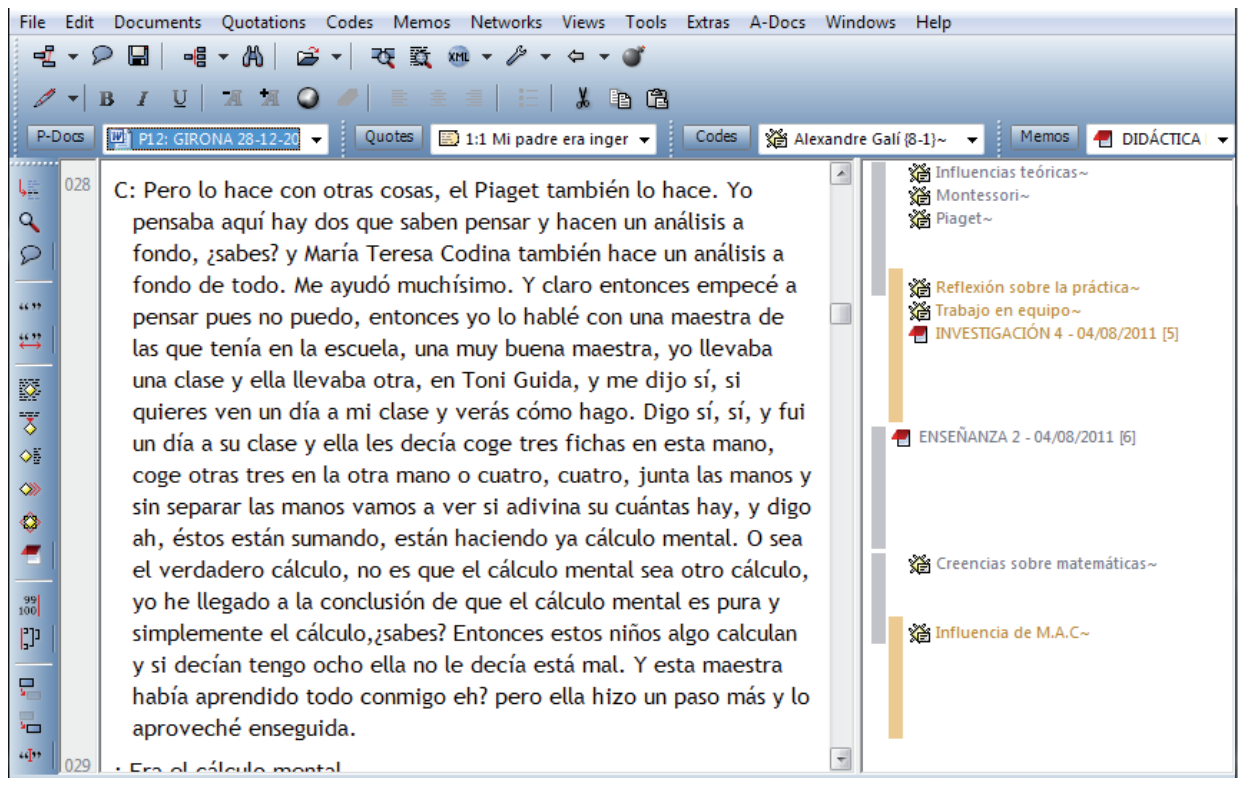

El programa también permite hacer anotaciones en relación con alguna parte del texto, en donde guardar ideas que van surgiendo desde esta primera toma de contacto con todo el material recopilado, y que más adelante se incorporan para el análisis.

En esta fase de codificación abierta se obtiene un listado de códigos extenso, como el que aparece en la Imagen 2 (se trata de una captura de pantalla de nuestro ejemplo, en la que aparece algo menos de un tercio del listado completo de códigos). Observando la segunda columna por la izquierda, vemos que hay códigos con una sola aparición (Cuisenaire) y otro que llega hasta 251 (Forma de ser). 
IMAGEN 2

PANTAlLa DEL GeSTOR DE CÓDigos DE ATLAS.TI

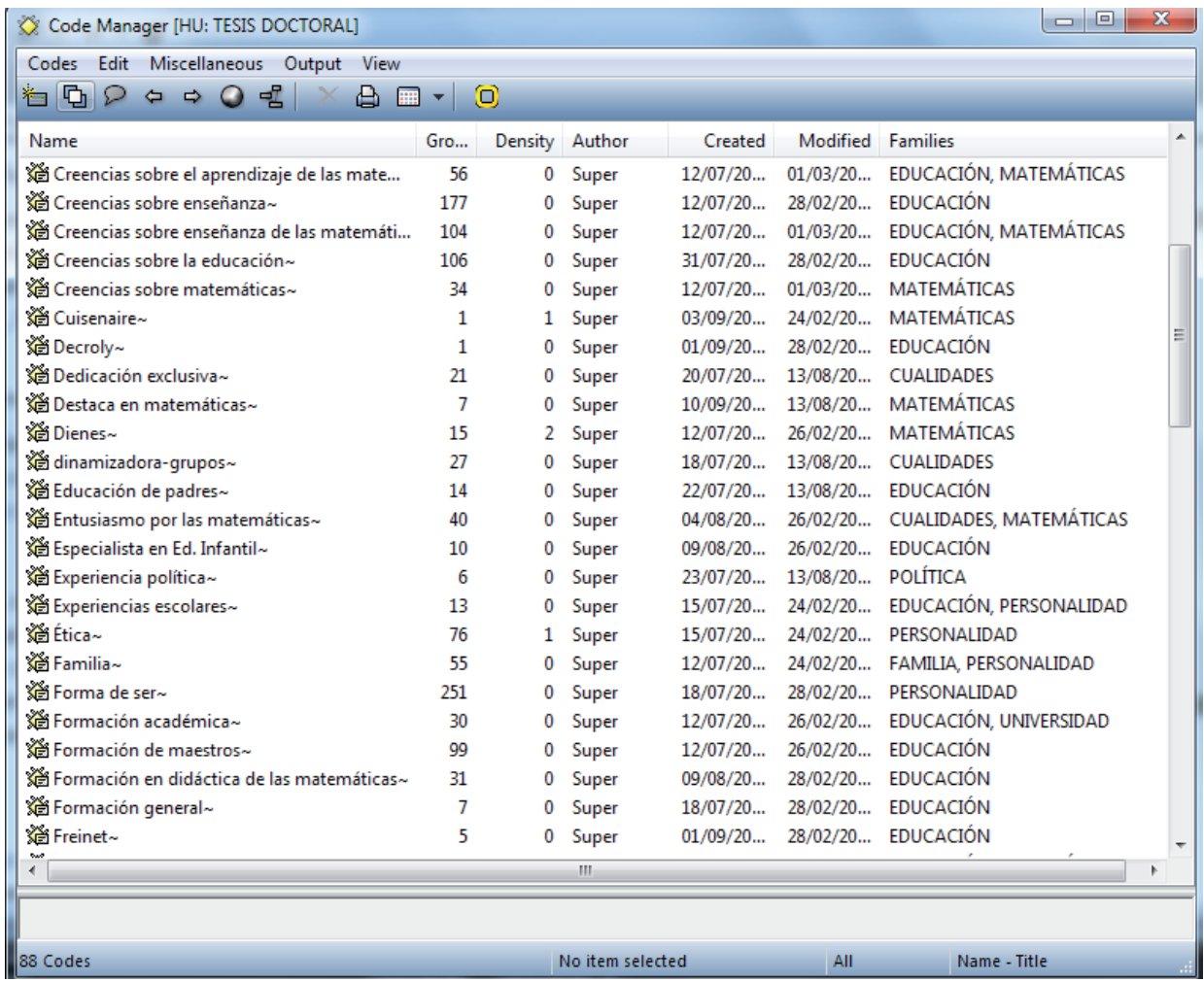

En esa misma pantalla, en la columna de la derecha, figuran lo que se denomina familias de códigos, con la denominación que le hemos asignado a cada una de ellas. En este caso son ocho, una para cada categoría que se estableció al principio. 
MARÍA SOTOS SERRANO, CARMEN LÓPEZ ESTEBAN Y ANA BELÉN SÁNCHEZ GARCÍA

IMAGEN 3

PANTALla DEL GESTOR DE FAMILIAS DE CÓDIGOS DE ATLAS.TI

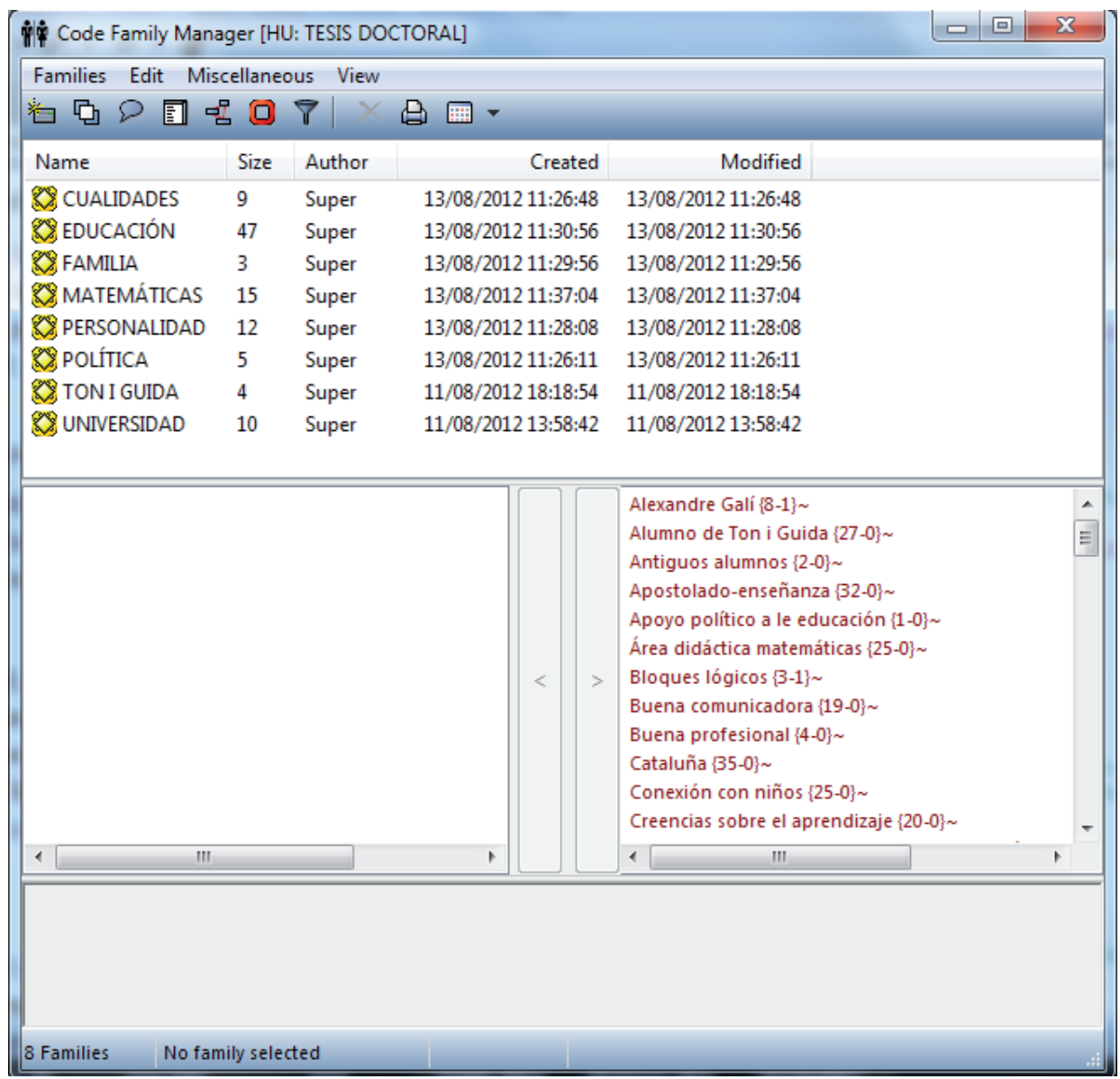

A partir de todo este trabajo, se procede a la fase de agrupación, que consiste en generar un documento de texto para cada una de las familias de códigos, en el que sólo se incluyen todos los fragmentos de texto pertenecientes a cada familia. Y una vez agrupados se pasa a la fase de integración local, en donde se comienza el trabajo sistemático de análisis e interpretación de cada una de las familias y se formulan las categorías conceptuales que mejor representan a todos los códigos. En la Tabla 1 hemos resumido las fases de una de las categorías con que contábamos, la del carácter personal de M. Antònia. Para esa categoría utilizamos la familia de códigos personalidad, y en ella establecemos siete categorías conceptuales 
(solidaridad, obstinada, creativa, cooperación, sensibilidad, fortaleza e innovadora), que cubren exhaustivamente todo el campo semántico de dicha familia.

Finalmente, en la fase de codificación selectiva, de lo que se trata es de descubrir las categorías centrales que están presentes a lo largo del relato de vida, qué relaciones mantienen entre ellas y qué papel desempeñan el resto de categorías en dicha secuencia. Para ello, cada fragmento de texto seleccionado vuelve a su lugar de origen, para descubrir las relaciones que puedan existir entre las diferentes categorías, así como para definir las categorías centrales (en este caso son dos, independencia y compromiso).

Así, el análisis conjunto del relato de vida (la totalidad de las categorías) permite descubrir mediante qué categorías centrales se puede comprender todo el relato de vida, especialmente en los momentos de la trayectoria de M. Antònia que han supuesto cambios importantes.

TABLA 1

CATEGORÍA CARÁCTER PERSONAL CON SUS DISTINTAS SUBCATEGORÍAS

\begin{tabular}{|c|c|c|c|}
\hline \multirow[b]{2}{*}{ CATEGORÍAS } & \multicolumn{3}{|c|}{ SUBCATEGORÍAS SEGÚN FASES DEL ANÁLISIS } \\
\hline & CODIFICACIÓN ABIERTA & INTEGRACIÓN LOCAL & $\begin{array}{c}\text { CODIFICACIÓN } \\
\text { SELECTIVA (CATEGORÍAS } \\
\text { CENTRALES) } \\
\end{array}$ \\
\hline \multirow{7}{*}{ Carácter personal } & \multirow{7}{*}{ Personalidad } & Innovadora & \multirow{3}{*}{ Independencia } \\
\hline & & Obstinada & \\
\hline & & Creativa & \\
\hline & & Cooperación & \multirow{4}{*}{ Compromiso } \\
\hline & & Sensibilidad & \\
\hline & & Fortaleza & \\
\hline & & Solidaridad & \\
\hline Entorno familiar & Familia & & \\
\hline $\begin{array}{l}\text { Cualidades } \\
\text { personales }\end{array}$ & Cualidades & & \\
\hline $\begin{array}{c}\text { Escuela } \\
\text { (alumna y maestra) }\end{array}$ & Educación & & \\
\hline Matemáticas & Matemáticas & & \\
\hline Universidad & Universidad & & \\
\hline Contexto político & Política & & \\
\hline Escola Ton $i$ Guida & Ton i Guida & & \\
\hline
\end{tabular}

En este caso, pensamos que las mejores categorías centrales para comprender la trayectoria biográfica de M. Antònia son las relacionadas con el carácter personal (independencia y compromiso) y con el entorno profesional (matemáticas y educación), pudiendo establecer con ellas un espacio bidimensional en el que poder 
MARÍA SOTOS SERRANO, CARMEN LÓPEZ ESTEBAN Y ANA BELÉN SÁNCHEZ GARCÍA

ubicar los momentos de cambios y decisiones que se producen en la biografía de M. Antònia (Sotos, 2015, 128).

\section{CONCLUSIONES, LIMITACIONES Y PROSPECTIVAS}

En primer lugar, mantenemos la hipótesis inicial acerca de la pertinencia de la perspectiva biográfica como metodología para el análisis de la trayectoria profesional de cualquier docente. Mediante dicha perspectiva, junto al uso de la entrevista abierta como técnica de investigación social, se construyen relatos de vida en los que juegan un papel importante los elementos actitudinales de cada sujeto.

Además, la elaboración del relato de vida sirve para la verbalización del proceso de construcción del saber pedagógico a partir de los saberes prácticos de cada docente. En este sentido, el propio proceso investigador, en el que se establece una estrecha colaboración entre sujeto investigador y sujeto investigado, es también un espacio de intercambio creativo y de reflexión sobre la práctica docente.

En el terreno más estrictamente metodológico, el proceso de análisis del discurso que hemos utilizado, que sigue las cuatro fases recomendadas por Weiss (1994), permite obtener categorías analíticas que posibilitan comprender toda la trayectoria pedagógica y, consiguientemente, se han podido interpretar muchos de los significados que la propia docente le da a su conducta pedagógica, como también los objetos matemáticos que servían de mediadores en los contextos didácticos y sus implicaciones para la enseñanza de las matemáticas. Todo lo anterior se consigue a partir de la información recogida en las entrevistas abiertas y mediante la propia rutina analítica que comienza con la codificación abierta y concluye con la codificación selectiva.

Finalmente, conviene añadir algunas consideraciones acerca de los criterios de calidad de las investigaciones realizadas mediante técnicas cualitativas.

Resulta necesaria la continua mejora de los estándares de calidad de cualquier tipo de investigación, y coincidimos en el planteamiento de quienes proponen criterios de calidad específicos para las investigaciones cualitativas. Si los planteamientos epistemológicos y metodológicos son diferentes, parece sensato que también se diferencien en los criterios de calidad aplicados. Siguiendo la propuesta de Erlandson y otros (1993), Valles establece una correspondencia entre los criterios de validez cuantitativos y los cualitativos. En principio los criterios de referencia son los mismos: veracidad, generalización y consistencia. Estos tres criterios se consiguen, en las investigaciones cuantitativas, mediante los criterios de validez interna, validez externa y fiabilidad; mientras que en las investigaciones cualitativas se obtienen mediante los criterios de credibilidad, transferibilidad y dependibilidad:

La credibilidad de un estudio cualitativo se relaciona con el uso que se haya hecho de un conjunto de recursos técnicos (duración e intensidad de la observación-participación en el contexto estudiado; triangulación de datos, métodos e investigadores; acopio de documentación escrita, visual propia del contexto; discusiones con 
colegas; revisiones de información e interpretación con las personas estudiadas; registro de cuadernos de campo y diarios de investigación) [...]. La transferibilidad se logra, sobre todo, a través de los diversos procedimientos de muestreo cualitativo, en contraposición a los procedimientos cuantitativos de muestreo probabilístico. Mientras que la dependibilidad (dependibility) se hace operativa mediante una suerte de auditoría externa. Para ello el investigador cualitativo debería facilitar la documentación que haga posible tal inspección (Valles, 1997, 104).

En nuestro caso, la credibilidad queda garantizada por la triangulación propia de los relatos de vida, las discusiones con colegas y la revisión del trabajo con la persona estudiada. La transferibilidad no es un aspecto estrictamente necesario cuando se realiza un estudio de caso único, ya que la pretensión no es analizar coincidencias entre casos diferentes para poder generalizar conclusiones. Y la dependibilidad se obtiene cuando se someten los resultados, así como los datos analizados, al criterio de jueces reconocidos, por eso acompañamos a la publicación del informe todos los datos primarios con los que se ha trabajado.

Junto a esos tres criterios, en las investigaciones mediante historias de vida hay que tener siempre en consideración las cuestiones éticas, y la mejor garantía ética es la de establecer un proceso de validación con el sujeto de dicha historia (Measor y Sikes, 2004). En nuestro caso, hemos compartido con M. Antònia Canals todos los resultados de nuestro análisis, y hemos discutido con ella numerosas cuestiones. En algunas ocasiones eso ha servido para corregir algunas interpretaciones equivocadas, aunque en otras no hemos llegado a un acuerdo completo, pero ambas partes hemos aceptado esas discrepancias. Cuando un sujeto le cede a otro el relato de su vida, el resultado final de esa cesión ha de ser gratificante para ambas.

\section{REFERENCIAS BIBLIOGRÁFICAS}

Aguilar, J. M. (1999) Entrevista en profundidad. Buenos Aires, Lumen/Humanitas.

ARnAus, R. (1993) Vida professional y acció pedagógica. A la recerca de la comprensió d'una mestra. Un estudi de cas. Tesis doctoral inédita. Barcelona, Departamento de Didáctica y Organización Escolar, Universidad de Barcelona.

Balan, J. (comp.) (1974) Las historias de vida en ciencias sociales. Buenos Aires, Nueva Visión.

Bertaux, D. (2005) Los relatos de vida. Perspectiva etnosociológica. Barcelona, Bellaterra.

CASEY, K. (2004) ¿Por qué abandonan la docencia las mujeres activistas progresistas?: teoría, metodología y política en la investigación sobre las historias de vida, en GoOdson, I. F. (ed.) Historias de vida del profesorado. Barcelona, Octaedro, 245-268.

Connelly, F. M. y Clandinin, D. J. (1986) On Narrative Method, Personal Philosophy, and Narrative Unities in the Story of Teaching. Journal of Research in Science Teaching, 23 (4), 293-310.

CONTRERAS, J. (1985) ¿El pensamiento o el conocimiento del profesor? Una crítica a los postulados de las investigaciones sobre el pensamiento del profesor y sus implicaciones para la formación del profesorado. Revista de Educación, 277, 5-28.

DenzIn, N. K. (1970) The research act. Chicago, Aldine. 
DíAz, V. (2005) Teoría emergente en la construcción del saber pedagógico. Revista Iberoamericana de Educación, 37/3. http://cort.as/EE63.

Erlandson, D. A.; Harris, E. L.; Skipper, B. L. y Allen, S. D. (1993) Doing Naturalistic Inquiry. Londres, Sage.

Goodson, I. F. (ed.) (2004) Historias de vida del profesorado. Barcelona, Octaedro.

HERnÁNDEZ, F. (ed.) (2004) Las historias de vida como estrategia de visibilización y generación de saber pedagógico, en Goodson, I. F. (ed.) Historias de vida del profesorado. Barcelona, Octaedro, 9-26.

JANESICK, V. J. (1982) Making sense of classroom group processes through a case study. Curriculum Inquiry, vol. XII, 12, 178-194.

Kohl, M. (1993) Biografía: relato, texto, método, en Marinas, J. M. y SANTAMarina, C. (eds.) La Historia oral: métodos y experiencias. Madrid, Debate, 173-184.

LARSSON, S. (1984) Describing teachers' conception of their professional world, en HaLKes, R. y Olson, J. K. (eds.) Teacher thinking. A new perspective on persisting problems in education. Lisse, Swets \& Zeitlinger, 35-50.

MEASOR, L. y SIKES, P. (2004) Una visita a las historia de vida: ética y metodología de la historia de vida, en Goodson, I. F. (ed.) Historias de vida del profesorado. Barcelona, Octaedro, 269-295.

PÉREZ, A. (1985) Paradigmas contemporáneos de investigación didáctica, en GimenO, J. y PÉReZ, A. (eds.) La enseñanza: su teoría y su práctica. Madrid, Akal, 95-138.

Plasencia, I. del C.; Güemes, R. M.; DorTA, J. A. y Espinel, M. C. (1999) Metodología utilizada en un trabajo sobre visualización matemática. Revista de Investigación Educativa, 14 (1), $167-185$.

Sotos, M. (2015) Didáctica de las matemáticas y desarrollo profesional de una maestra. El caso de Maria Antònia Canals $i$ Tolosa. Tesis doctoral inédita. Salamanca, Departamento de Didáctica de las Matemáticas y CCEE, Universidad de Salamanca.

TARDIF, M. (2004) Los saberes del docente y su desarrollo profesional. Madrid, Narcea.

TRINIDAD, A.; CARRERO, V. y SORIANO, R. M. (2006) Teoría fundamentada "Grounded Theory». La construcción de la teoría a través del análisis interpretacional. Madrid, Centro de Investigaciones Sociológicas.

Valles, M. S. (1997) Técnicas cualitativas de investigación. Reflexión metodológica y práctica profesional. Madrid, Síntesis.

WEISS, R. (1994) Learning for strangers. The art and method of qualitative interview studies. New York, The Free Press. 